\title{
Generalized Jensen-Mercer Inequality for Functions with Nondecreasing Increments
}

\author{
Asif R. Khan ${ }^{1,2}$ and Sumayyah Saadi ${ }^{1,2}$ \\ ${ }^{1}$ Institute of Business Administration, University Road, Karachi 75270, Pakistan \\ ${ }^{2}$ Department of Mathematics, Faculty of Science, University of Karachi, University Road, Karachi 75270, Pakistan \\ Correspondence should be addressed to Sumayyah Saadi; sumayyahsaadi@hotmail.com
}

Received 25 February 2016; Accepted 3 April 2016

Academic Editor: Wing-Sum Cheung

Copyright (c) 2016 A. R. Khan and S. Saadi. This is an open access article distributed under the Creative Commons Attribution License, which permits unrestricted use, distribution, and reproduction in any medium, provided the original work is properly cited.

In the year 2003, McD Mercer established an interesting variation of Jensen's inequality and later in 2009 Mercer's result was generalized to higher dimensions by M. Niezgoda. Recently, Asif et al. has stated an integral version of Niezgoda's result for convex functions. We further generalize Niezgoda's integral result for functions with nondecreasing increments and give some refinements with applications. In the way, we generalize an important result, Jensen-Boas inequality, using functions with nondecreasing increments. These results would constitute a valuable addition to Jensen-type inequalities in the literature.

\section{Introduction and Preliminaries}

Let us start with Jensen's inequality for convex functions, one of the most celebrated inequalities in mathematics and statistics (for detailed discussion and history, see $[1,2]$ ). Throughout the paper, we assume that $J$ and $[a, b]$ are intervals in $\mathbb{R}$, and $\mathbf{J}$ is an interval in $\mathbb{R}^{k}$ and $\mathbf{U} \subset \mathbb{R}^{k}$ is a $k$-dimensional rectangle for integer $k \geq 1$. Also for weights $w_{i}, i \in\{1, \ldots, n\}$, we would use $W_{n}=\sum_{i=1}^{n} w_{i}$.

Proposition 1. Let $x_{1}, x_{2}, \ldots, x_{n} \in[a, b]$ and let $w_{1}, w_{2}, \ldots$, $w_{n}$ be nonnegative real numbers such that $W_{n}>0$. If $\varphi$ : $[a, b] \rightarrow \mathbb{R}$ is a convex function, then the following inequality holds:

$$
\varphi\left(\frac{1}{W_{n}} \sum_{i=1}^{n} w_{i} x_{i}\right) \leq \frac{1}{W_{n}} \sum_{i=1}^{n} w_{i} \varphi\left(x_{i}\right)
$$

In [3], McD Mercer proved the following variant of Jensen's inequality, which we will refer to as Mercer's inequality.
Proposition 2. Under the assumptions of Proposition 1, the following inequality holds:

$$
\begin{aligned}
& \varphi\left(m_{1}+m_{2}-\frac{1}{W_{n}} \sum_{i=1}^{n} w_{i} x_{i}\right) \\
& \quad \leq \varphi\left(m_{1}\right)+\varphi\left(m_{2}\right)-\frac{1}{W_{n}} \sum_{i=1}^{n} w_{i} \varphi\left(x_{i}\right),
\end{aligned}
$$

where

$$
\begin{aligned}
& m_{1}=\min _{1 \leq i \leq n}\left\{x_{i}\right\}, \\
& m_{2}=\max _{1 \leq i \leq n}\left\{x_{i}\right\} .
\end{aligned}
$$

There are many versions, variants, and generalizations of Propositions 1 and 2; see for example [4-7]. Here we state an integral version of Jensen's inequality from [1, pages 58-59] which will be needed in our main result.

Proposition 3. Let $f:[a, b] \rightarrow J$ be a continuous function. If the function $H:[a, b] \rightarrow \mathbb{R}$ is nondecreasing and bounded 
and $H(a) \neq H(b)$, then for every continuous convex function $\varphi: J \rightarrow \mathbb{R}$ the inequality

$$
\varphi\left(\frac{\int_{a}^{b} f(t) d H(t)}{\int_{a}^{b} d H(t)}\right) \leq \frac{\int_{a}^{b} \varphi(f(t)) d H(t)}{\int_{a}^{b} d H(t)}
$$

holds.

In our construction for next proposition, we recall the definitions of majorization.

For fixed $n \geq 2$,

$$
\begin{aligned}
& \mathbf{x}=\left(x_{1}, \ldots, x_{n}\right), \\
& \mathbf{y}=\left(y_{1}, \ldots, y_{n}\right),
\end{aligned}
$$

denote two real $n$-tuples and let

$$
\begin{aligned}
& x_{[1]} \geq x_{[2]} \geq \cdots \geq x_{[n]}, \\
& y_{[1]} \geq y_{[2]} \geq \cdots \geq y_{[n]}
\end{aligned}
$$

be their ordered components.

Definition 4. For $\mathbf{x}, \mathbf{y} \in \mathbb{R}^{n}$,

$$
\mathbf{x} \prec \mathbf{y} \quad \text { if }\left\{\begin{array}{l}
\sum_{i=1}^{k} x_{[i]} \leq \sum_{i=1}^{k} y_{[i]}, \quad k \in\{1, \ldots, n-1\}, \\
\sum_{i=1}^{n} x_{[i]}=\sum_{i=1}^{n} y_{[i]} ;
\end{array}\right.
$$

when $\mathbf{x} \prec \mathbf{y}, \mathbf{x}$ is said to be majorized by $\mathbf{y}$ or $\mathbf{y}$ majorizes $\mathbf{x}$.

This notion and notation of majorization were introduced by Hardy et al. in [8].

The following extension of inequality (2) was given by Niezgoda in [6] which is referred to as Niezgoda's inequality.

Proposition 5. Let $\varphi:[a, b] \rightarrow \mathbb{R}$ be a continuous convex function. Suppose that $\alpha=\left(\alpha_{1}, \ldots, \alpha_{m}\right)$ with $\alpha_{j} \in[a, b]$ and $\mathbf{X}=\left(x_{i j}\right)$ is a real $n \times m$ matrix such that $x_{i j} \in[a, b]$ for all $i \in\{1, \ldots, n\}$ and $j \in\{1, \ldots, m\}$.

If $\alpha$ majorizes each row of $\mathbf{X}$, that is,

$$
\begin{aligned}
& \mathbf{x}_{i .}=\left(x_{i 1}, \ldots, x_{i m}\right) \prec\left(\alpha_{1}, \ldots, \alpha_{m}\right)=\alpha \\
& \qquad \text { for each } i \in\{1, \ldots, n\},
\end{aligned}
$$

then we have the inequality

$$
\begin{aligned}
\varphi\left(\sum_{j=1}^{m} \alpha_{j}-\frac{1}{W_{n}} \sum_{j=1}^{m-1} \sum_{i=1}^{n} w_{i} x_{i j}\right) \\
\leq \sum_{j=1}^{m} \varphi\left(\alpha_{j}\right)-\frac{1}{W_{n}} \sum_{j=1}^{m-1} \sum_{i=1}^{n} w_{i} \varphi\left(x_{i j}\right),
\end{aligned}
$$

where $W_{n}>0$ with nonnegative weights $w_{i}$.
The present paper is organized as follows: after some preliminaries, in Section 2, we recall definition of functions with nondecreasing increments and their properties and note that some inequalities from Section 1 which held true for convex functions also hold for functions with nondecreasing increments. In Section 3, we give an integral generalization of Niezgoda's inequality. In the process, we will use an integral majorization result of Pečarić [9] and prove a result which gives the Jensen-Boas inequality on disjoint set of subintervals for functions with nondecreasing increments. In Section 4, we will discuss some refinements of the main results we proved in Section 3. The last part of this section is devoted to the applications of some related results.

\section{Introduction to Functions with Nondecreasing Increments}

In 1964, Brunk defined an interesting class of multivariate real valued functions [10] known as functions with nondecreasing increments.

Definition 6. A real valued function $f$ on a $k$-dimensional rectangle $\mathbf{U} \subset \mathbb{R}^{k}$, where $k$ is a fixed positive integer, is said to have nondecreasing increments if

$$
\begin{aligned}
& f(\mathbf{a}+\mathbf{h})-f(\mathbf{a}) \leq f(\mathbf{b}+\mathbf{h})-f(\mathbf{b}) \\
& \text { whenever } \mathbf{0} \leq \mathbf{h} \in \mathbb{R}^{k}, \mathbf{a} \leq \mathbf{b}, \mathbf{a}, \mathbf{b}+\mathbf{h} \in \mathbf{U},
\end{aligned}
$$

where partial order is defined by $\left(x_{1}, x_{2}, \ldots, x_{k}\right) \leq\left(y_{1}, y_{2}\right.$, $\left.\ldots, y_{k}\right) \Leftrightarrow x_{1} \leq y_{1}, \ldots, x_{k} \leq y_{k}$.

In the same paper [10], Brunk gave some examples and properties of the functions which we discuss below.

\subsection{Examples of Functions with Nondecreasing Increments}

(i) The simplest example of a function with nondecreasing increments is a constant function.

(ii) Lines of the form $\mathbf{x}=\mathbf{a} t+\mathbf{b}$, where $(0, \ldots, 0) \leq$ $\mathbf{a} \in \mathbb{R}^{k}$ and $\mathbf{b} \in \mathbb{R}^{k}$ whose direction cosines are nonnegative, also belong to the family of functions with nondecreasing increments.

(iii) An important continuous function with nondecreasing increments is $\phi: \mathbb{R}^{2} \rightarrow \mathbb{R}$ defined by $\phi(x, y)=$ $x y$ :

Another useful continuous function with nondecreasing increments is $\varphi:[0, \infty)^{k} \rightarrow \mathbb{R}$ defined by $\varphi(\mathbf{x})=\prod_{i=1}^{k} x_{i}$.

(iv) An interesting and widely used example of such functions is the Cauchy functional equation

$$
F(\mathbf{x}+\mathbf{y})=F(\mathbf{x})+F(\mathbf{y}) .
$$

2.2. Properties of Functions with Nondecreasing Increments. Functions with nondecreasing increments possess the following properties: 
(i) A function with nondecreasing increments is not necessarily continuous.

(ii) If the first partial derivatives of a function $f: \mathbf{U} \rightarrow \mathbb{R}$ exist for $\mathbf{x} \in \mathbf{U}$, then $\mathbf{x}$ has nondecreasing increments if and only if each of these partial derivatives is nondecreasing in each argument.

(iii) If the second partial derivatives of a function $f: \mathbf{U} \rightarrow$ $\mathbb{R}$ exist for $\mathbf{x} \in \mathbf{U}$, then $\mathbf{x}$ has nondecreasing increments if and only if each of these partial derivatives is nonnegative.

(iv) If a function $f$ with nondecreasing increments is continuous for $\mathbf{b} \leq \mathbf{x} \leq \mathbf{a}+\mathbf{b}$, where $\mathbf{0} \leq \mathbf{a} \in \mathbb{R}^{k}$, then the function $\varphi:[0,1] \rightarrow \mathbb{R}$ defined by $\varphi(t)=f(t \mathbf{a}+\mathbf{b})$ is convex.

We define here a special type of functions which belong to the class of functions with nondecreasing increments and which themselves contain the class of convex functions. These functions are called Wright convex functions [1, page 7].

Definition 7. We say $f: I \rightarrow \mathbb{R}$ is a Wright convex function if $\forall a, b+h \in I$ with $a<b$ and $h>0$ we have

$$
f(a+h)-f(a) \leq f(b+h)-f(b) .
$$

Remark 8. It is easy to see that, in one-dimensional case, functions with nondecreasing increments are Wright convex functions. Also, continuous Wright convex functions are convex functions. Thus, the class of convex functions is a proper subclass of the Wright convex functions.

Now we state some results that will be needed to derive our main results. The following proposition gives Jensen's inequality for functions with nondecreasing increments [11].

Proposition 9. Let $f: \mathbf{U} \rightarrow \mathbb{R}$ be a continuous function with nondecreasing increments, let $\mathbf{w}$ be a nonnegative $n$-tuple such that $W_{n}>0$, and let $\mathbf{x}^{(i)} \in \mathbf{U}$, where $i \in\{1, \ldots, n\}$, be such that $\mathbf{x}^{(1)} \leq \cdots \leq \mathbf{x}^{(n)}$ or $\mathbf{x}^{(1)} \geq \cdots \geq \mathbf{x}^{(n)}$. Then, it holds that

$$
f\left(\frac{1}{W_{n}} \sum_{i=1}^{n} w_{i} \mathbf{x}^{(i)}\right) \leq \frac{1}{W_{n}} \sum_{i=1}^{n} w_{i} f\left(\mathbf{x}^{(i)}\right) .
$$

We now state Jensen-Steffensen's inequality for functions with nondecreasing increments [12].

Proposition 10. $\mathbf{f}:[a, b] \rightarrow \mathbf{J}$ is a nondecreasing continuous function and $H:[a, b] \rightarrow \mathbb{R}$ is of bounded variation satisfying

$$
H(a) \leq H(t) \leq H(b) \quad \forall t \in[a, b], H(b)>H(a) .
$$

If $\varphi: \mathbf{J} \rightarrow \mathbb{R}$ is a continuous function with nondecreasing increments, then the following inequality holds:

$$
\varphi\left(\frac{\int_{a}^{b} \mathbf{f}(t) d H(t)}{\int_{a}^{b} d H(t)}\right) \leq \frac{\int_{a}^{b} \varphi(\mathbf{f}(t)) d H(t)}{\int_{a}^{b} d H(t)},
$$

where $\int_{a}^{b} \mathbf{f} d H=\left(\int_{a}^{b} f_{1} d H, \ldots, \int_{a}^{b} f_{k} d H\right)$.
At this stage, we prove Jensen-Boas inequality for functions with nondecreasing increments as follows.

Theorem 11. Let $\mathbf{f}:[a, b] \rightarrow \mathbf{J}$ be a continuous and monotonic (either nonincreasing or nondecreasing) map in each of the $k$ intervals $\left(b_{i-1}, b_{i}\right)$. Let $H:[a, b] \rightarrow \mathbb{R}$ be continuous or of bounded variation satisfying

$$
\begin{aligned}
H(a) & \leq H\left(a_{1}\right) \leq H\left(b_{1}\right) \leq H\left(a_{2}\right) \leq \cdots \leq H\left(b_{k-1}\right) \\
& \leq H\left(a_{k}\right) \leq H(b)
\end{aligned}
$$

for all $a_{i} \in\left(b_{i-1}, b_{i}\right)\left(b_{0}=a, b_{k}=b\right)$ and $H(b)>H(a)$. If $\varphi$ is a continuous function having nondecreasing increments in each of the $k$ intervals $\left(b_{i-1}, b_{i}\right)$, then we have the following inequality:

$$
\varphi\left(\frac{\int_{a}^{b} \mathbf{f}(t) d H(t)}{\int_{a}^{b} d H(t)}\right) \leq \frac{\int_{a}^{b} \varphi(\mathbf{f}(t)) d H(t)}{\int_{a}^{b} d H(t)} .
$$

Proof. Using Jensen's inequality (13) for nonnegative $n$-tuple $\mathbf{w}$ and Jensen-Steffensen's inequality (15), if $H(a) \leq H\left(a_{1}\right) \leq$ $H\left(b_{1}\right) \leq H\left(a_{2}\right) \leq \cdots \leq H\left(b_{k-1}\right) \leq H(b)$ we have

$$
\varphi\left(\frac{\int_{b_{i-1}}^{b_{i}} \mathbf{f}(t) d H(t)}{\int_{b_{i-1}}^{b_{i}} d H(t)}\right) \leq \frac{\int_{b_{i-1}}^{b_{i}} \varphi(\mathbf{f}(t)) d H(t)}{\int_{b_{i-1}}^{b_{i}} d H(t)}
$$

for $i \in\{1,2, \ldots, k\}$.

If we consider $t_{i}=\left(\int_{b_{i-1}}^{b_{i}} \mathbf{f}(t) d H(t) / \int_{b_{i-1}}^{b_{i}} d H(t)\right)$ and $w_{i}=$ $\int_{b_{i-1}}^{b_{i}} d H(t)$, then we can write

$$
\varphi\left(t_{i}\right) \leq \frac{1}{w_{i}} \int_{b_{i-1}}^{b_{i}} \varphi(\mathbf{f}(t)) d H(t)
$$

using this fact, we have

$$
\begin{gathered}
\varphi\left(\frac{\int_{a}^{b} \mathbf{f}(t) d H(t)}{\int_{a}^{b} d H(t)}\right)=\varphi\left(\frac{\sum_{i=1}^{n} w_{i} t_{i}}{\sum_{i=1}^{n} w_{i}}\right) \\
\leq \frac{\sum_{i=1}^{n} w_{i} \varphi\left(t_{i}\right)}{\sum_{i=1}^{n} w_{i}} \\
\leq \frac{\sum_{i=1}^{n} w_{i}\left(1 / w_{i}\right) \int_{b_{i-1}}^{b_{i}} \varphi(\mathbf{f}(t)) d H(t)}{\sum_{i=1}^{n} w_{i}} \\
=\frac{\int_{a}^{b} \varphi(\mathbf{f}(t)) d H(t)}{\int_{a}^{b} d H(t)} .
\end{gathered}
$$

The following proposition represents an integral majorization result which would be needed in our next main result [1, page 328 ]. 
Proposition 12. Let $\mathbf{f}, \mathbf{g}:[a, b] \rightarrow \mathbf{J}$ be two nonincreasing continuous functions and let $H:[a, b] \rightarrow \mathbb{R}$ be a function of bounded variation. If

$$
\begin{aligned}
& \int_{a}^{x} \mathbf{f}(t) d H(t) \leq \int_{a}^{x} \mathbf{g}(t) d H(t), \\
& \quad \text { for each } x \in(a, b), \\
& \int_{a}^{b} \mathbf{f}(t) d H(t)=\int_{a}^{b} \mathbf{g}(t) d H(t)
\end{aligned}
$$

hold, then for every continuous function with nondecreasing increments $\varphi: \mathbf{J} \rightarrow \mathbb{R}$ the following inequality holds:

$$
\int_{a}^{b} \varphi(\mathbf{f}(t)) d H(t) \leq \int_{a}^{b} \varphi(\mathbf{g}(t)) d H(t) .
$$

Remark 13. If $\mathbf{f}, \mathbf{g}:[a, b] \rightarrow \mathbf{J}$ are two nondecreasing continuous functions such that

$$
\begin{aligned}
& \int_{x}^{b} \mathbf{f}(t) d H(t) \leq \int_{x}^{b} \mathbf{g}(t) d H(t), \\
& \quad \text { for each } x \in(a, b), \\
& \int_{a}^{b} \mathbf{f}(t) d H(t)=\int_{a}^{b} \mathbf{g}(t) d H(t),
\end{aligned}
$$

then again inequality (22) holds. In this paper, we will state our results for nonincreasing $\mathbf{f}$ and $\mathbf{g}$ satisfying the assumption of Proposition 12, but they are still valid for nondecreasing $\mathbf{f}$ and $\mathbf{g}$ satisfying the above condition (see, e.g., [13, page 584]).

\section{Generalized Jensen-Mercer Inequality}

Here we state a result needed in the main theorems of this section. The following lemma shows that the subintervals in the Jensen-Boas inequality (see Theorem 11) can be disjoint for the inequality of type (15) to hold.

Lemma 14. Let $H:[a, b] \rightarrow \mathbb{R}$ be continuous or a function of bounded variation and let $a \leq a_{1} \leq b_{1} \leq a_{2} \leq \cdots \leq a_{k} \leq$ $b_{k} \leq b$ be a partition of the interval $[a, b] ; I=\bigcup_{i=1}^{k}\left[a_{i}, b_{i}\right]$ and $L=\int_{I} d H(t)>0$. If

$$
H\left(a_{i}\right) \leq H(t) \leq H\left(b_{i}\right) \quad \forall t \in\left(a_{i}, b_{i}\right), 1 \leq i \leq k,
$$

then, for every function $\mathbf{f}:[a, b] \rightarrow \mathbf{J}$ which is continuous and monotonic (either nonincreasing or nondecreasing) in each of the $k$ intervals $\left(a_{i}, b_{i}\right)$ and every continuous function with nondecreasing increments $\varphi: \mathrm{J} \rightarrow \mathbb{R}$, the following inequality holds:

$$
\varphi\left(\frac{1}{L} \int_{I} \mathbf{f}(t) d H(t)\right) \leq \frac{1}{L} \int_{I} \varphi(\mathbf{f}(t)) d H(t)
$$

Proof. Denote $w_{i}=\int_{a_{i}}^{b_{i}} d H(t)$. Due to (16), if $H\left(a_{i}\right)=H\left(b_{i}\right)$ then $d H$ is a null-measure on $\left[a_{i}, b_{i}\right]$ and $w_{i}=0$, while otherwise $w_{i}>0$. Denote $S=\left\{i: w_{i}>0\right\}$ and

$$
\mathbf{x}_{i}=\frac{1}{w_{i}} \int_{a_{i}}^{b_{i}} \mathbf{f}(t) d H(t), \quad \text { for } i \in S .
$$

Notice that

$$
\begin{array}{r}
L=\int_{I} d H(t)=\sum_{i \in S} w_{i}>0, \\
\int_{I} \varphi(\mathbf{f}(t)) d H(t)=\sum_{i \in S} \int_{a_{i}}^{b_{i}} \varphi(\mathbf{f}(t)) d H(t)
\end{array}
$$

and, due to Proposition 10,

$$
w_{i} \varphi\left(\mathbf{x}_{i}\right) \leq \int_{a_{i}}^{b_{i}} \varphi(\mathbf{f}(t)) d H(t), \quad \text { for } i \in S .
$$

Therefore, taking into account the discrete Jensen's inequality (13),

$$
\begin{aligned}
\varphi\left(\frac{1}{L} \int_{I} \mathbf{f}(t) d H(t)\right) & =\varphi\left(\frac{1}{L} \sum_{i \in S} w_{i} \mathbf{x}_{i}\right) \\
& \leq \frac{1}{L} \sum_{i \in S} w_{i} \varphi\left(\mathbf{x}_{i}\right) \\
& \leq \frac{1}{L} \sum_{i \in S} \int_{a_{i}}^{b_{i}} \varphi(\mathbf{f}(t)) d H(t) \\
& =\frac{1}{L} \int_{I} \varphi(\mathbf{f}(t)) d H(t) .
\end{aligned}
$$

The following theorem is our main result of this section and it gives a generalization of Proposition 5.

Theorem 15. Let $a=b_{0} \leq a_{1}<b_{1}<a_{2}<b_{2}<\cdots<a_{k}<b_{k} \leq$ $a_{k+1}=b, I=\bigcup_{i=1}^{k}\left(a_{i}, b_{i}\right), I^{c}=[a, b] \backslash I=\bigcup_{i=1}^{k+1}\left[b_{i-1}, a_{i}\right]$, and $H:[a, b] \rightarrow \mathbb{R}$ be a function of bounded variation such that $H\left(b_{i-1}\right) \leq H(t) \leq H\left(a_{i}\right)$ for all $t \in\left(b_{i-1}, a_{i}\right)$ and $1 \leq i \leq k+1$ and $L=\int_{I^{c}} d H(t)>0$.

Furthermore, let $(X, \Sigma, \mu)$ be a measure space with positive finite measure $\mu$, let $\mathbf{g}:[a, b] \rightarrow \mathbf{J}$ be a nonincreasing continuous function, and let $\mathbf{f}: X \times[a, b] \rightarrow \mathbf{J}$ be a measurable function such that the mapping $t \mapsto \mathbf{f}(s, t)$ is nonincreasing and continuous for each $s \in X$ :

$$
\int_{a}^{x} \mathbf{f}(s, t) d H(t) \leq \int_{a}^{x} \mathbf{g}(t) d H(t)
$$$$
\text { for each } x \in(a, b) \text {, }
$$

$\int_{a}^{b} \mathbf{f}(s, t) d H(t)=\int_{a}^{b} \mathbf{g}(t) d H(t)$ 
Then, for a continuous function with nondecreasing increments $\varphi: \mathrm{J} \rightarrow \mathbb{R}$, the following inequality holds:

$$
\begin{gathered}
\varphi\left(\frac { 1 } { L } \left(\int_{a}^{b} \mathbf{g}(t) d H(t)\right.\right. \\
\left.\left.-\frac{1}{\mu(X)} \int_{I} \int_{X} \mathbf{f}(s, t) d \mu(s) d H(t)\right)\right) \\
\leq \frac{1}{L}\left(\int_{a}^{b} \varphi(\mathbf{g}(t)) d H(t)-\frac{1}{\mu(X)}\right. \\
\left.\quad \int_{I} \int_{X} \varphi(\mathbf{f}(s, t)) d \mu(s) d H(t)\right) .
\end{gathered}
$$

Proof. Using Fubini's theorem, inequality (30), and Jensen's integral inequality (4), we have

$$
\begin{aligned}
& \varphi\left(\frac { 1 } { L } \left(\int_{a}^{b} \mathbf{g}(t) d H(t)\right.\right. \\
& \left.\left.-\frac{1}{\mu(X)} \int_{I} \int_{X} \mathbf{f}(s, t) d \mu(s) d H(t)\right)\right) \\
& =\varphi\left(\frac { 1 } { L } \left(\int_{a}^{b} \frac{1}{\mu(X)} \int_{X} \mathbf{g}(t) d H(t) d \mu(s)\right.\right. \\
& \left.\left.-\frac{1}{\mu(X)} \int_{I} \int_{X} \mathbf{f}(s, t) d \mu(s) d H(t)\right)\right)=\varphi\left(\frac{1}{\mu(X)}\right. \\
& \left.\quad \cdot \int_{X}\left[\frac{1}{L} \int_{I^{c}} \mathbf{f}(s, t) d H(t)\right] d \mu(s)\right) \leq \frac{1}{\mu(X)} \\
& \quad \cdot \int_{X} \varphi\left(\frac{1}{L} \int_{I^{c}} \mathbf{f}(s, t) d H(t)\right) d \mu(s) .
\end{aligned}
$$

Applying Lemma 14 and Proposition 12, respectively, we have

$$
\begin{aligned}
& \frac{1}{\mu(X)} \int_{X} \varphi\left(\frac{1}{L} \int_{I^{c}} \mathbf{f}(s, t) d H(t)\right) d \mu(s) \leq \frac{1}{\mu(X)} \\
& \cdot \int_{X}\left(\frac { 1 } { L } \left(\int_{a}^{b} \varphi(\mathbf{g}(t)) d H(t)\right.\right. \\
& \left.\left.-\int_{I} \varphi(\mathbf{f}(s, t)) d H(t)\right) d \mu(s)\right) \\
& =\frac{1}{L}\left(\frac{1}{\mu(X)} \int_{X} d \mu(s) \int_{a}^{b} \varphi(\mathbf{g}(t)) d H(t)-\frac{1}{\mu(X)}\right. \\
& \left.\cdot \int_{I} \varphi(\mathbf{f}(s, t)) d H(t) d \mu(s)\right) \\
& =\frac{1}{L}\left(\int_{a}^{b} \varphi(\mathbf{g}(t)) d H(t)-\frac{1}{\mu(X)}\right. \\
& \left.\cdot \int_{I} \int_{X} \varphi(\mathbf{f}(s, t)) d \mu(s) d H(t)\right) .
\end{aligned}
$$

The special case of Theorem 15 can be found in [14] which may be stated as follows.

Corollary 16. Let $a=b_{0} \leq a_{1}<b_{1}<a_{2}<b_{2}<\cdots<a_{k}<$ $b_{k} \leq a_{k+1}=b, I=\bigcup_{i=1}^{k}\left(a_{i}, b_{i}\right), I^{c}=[a, b] \backslash I=\bigcup_{i=1}^{k+1}\left[b_{i-1}, a_{i}\right]$, and $H:[a, b] \rightarrow \mathbb{R}$ be a function of bounded variation such that $H\left(b_{i-1}\right) \leq H(t) \leq H\left(a_{i}\right)$ for all $t \in\left(b_{i-1}, a_{i}\right)$ and $1 \leq i \leq$ $k+1$ and $L=\int_{I^{c}} d H(t)>0$.

Furthermore, let $(X, \Sigma, \mu)$ be a measure space with positive finite measure $\mu$, let $g:[a, b] \rightarrow J$ be a nonincreasing continuous function, and let $f: X \times[a, b] \rightarrow J$ be a measurable function such that the mapping $t \mapsto f(s, t)$ is nonincreasing and continuous for each $s \in X$ :

$$
\begin{aligned}
& \int_{a}^{x} f(s, t) d H(t) \leq \int_{a}^{x} g(t) d H(t) \\
& \qquad \text { for each } x \in(a, b), \\
& \int_{a}^{b} f(s, t) d H(t)=\int_{a}^{b} g(t) d H(t) .
\end{aligned}
$$

Then, for a continuous convex function $\varphi: J \rightarrow \mathbb{R}$, the following inequality holds:

$$
\begin{gathered}
\varphi\left(\frac { 1 } { L } \left(\int_{a}^{b} g(t) d H(t)\right.\right. \\
\left.\left.-\frac{1}{\mu(X)} \int_{I} \int_{X} f(s, t) d \mu(s) d H(t)\right)\right) \\
\leq \frac{1}{L}\left(\int_{a}^{b} \varphi(g(t)) d H(t)-\frac{1}{\mu(X)}\right. \\
\left.\quad \cdot \int_{I} \int_{X} \varphi(f(s, t)) d \mu(s) d H(t)\right) .
\end{gathered}
$$

\section{Refinements}

Let $(X, \Sigma, \mu)$ be a measure space with positive finite measure $\mu$. Throughout this section, we assume that $E \subset X$ with $\mu(E)$, $\mu\left(E^{c}\right)>0$ and we use the following notations:

$$
\begin{aligned}
W_{E} & =\frac{\mu(E)}{\mu(X)}, \\
W_{E^{c}} & =\frac{\mu\left(E^{c}\right)}{\mu(X)}=1-W_{E} .
\end{aligned}
$$


Theorem 17. Under the assumptions of Theorem 15, the following refinement is valid for every continuous function with nondecreasing increments $\varphi: \mathbf{J} \rightarrow \mathbb{R}:$

$$
\begin{aligned}
& \varphi\left(\frac { 1 } { L } \left(\int_{a}^{b} \mathbf{g}(t) d H(t)\right.\right. \\
& \left.\left.-\frac{1}{\mu(X)} \int_{I} \int_{X} \mathbf{f}(s, t) d \mu(s) d H(t)\right)\right) \leq F(\mathbf{f}, \mathbf{g}, \varphi ; \\
& E) \leq \frac{1}{L}\left(\int_{a}^{b} \varphi(\mathbf{g}(t)) d H(t)-\frac{1}{\mu(X)}\right. \\
& \left.\quad \cdot \int_{I} \int_{X} \varphi(\mathbf{f}(s, t)) d \mu(s) d H(t)\right),
\end{aligned}
$$

where

$$
\begin{gathered}
F(\mathbf{f}, \mathbf{g}, \varphi ; E)=W_{E} \varphi\left(\frac { 1 } { L } \left(\int_{a}^{b} \mathbf{g}(t) d H(t)\right.\right. \\
\left.\left.-\frac{1}{\mu(E)} \int_{I} \int_{E} \mathbf{f}(s, t) d \mu(s) d H(t)\right)\right) \\
+W_{E^{c}} \varphi\left(\frac { 1 } { L } \left(\int_{a}^{b} \mathbf{g}(t) d H(t)\right.\right. \\
\left.\left.-\frac{1}{\mu\left(E^{c}\right)} \int_{I} \int_{E^{c}} \mathbf{f}(s, t) d \mu(s) d H(t)\right)\right) .
\end{gathered}
$$

Proof. Using discrete Jensen's inequality (13) for functions with nondecreasing increments, we have

$$
\begin{aligned}
& \varphi( \frac{1}{L}\left(\int_{a}^{b} \mathbf{g}(t) d H(t)-\frac{1}{\mu(X)}\right. \\
&\left.\left.\cdot \int_{I} \int_{X} \mathbf{f}(s, t) d \mu(s) d H(t)\right)\right) \\
&=\varphi\left(W _ { E } \left[\frac { 1 } { L } \left(\int_{a}^{b} \mathbf{g}(t) d H(t)\right.\right.\right. \\
&\left.\left.-\frac{1}{\mu(E)} \int_{E} \int_{I} \mathbf{f}(s, t) d H(t)\right) d \mu(s)\right] \\
&+W_{E^{c}}\left[\frac { 1 } { L } \left(\int_{a}^{b} \mathbf{g}(t) d H(t)\right.\right. \\
&\left.\left.\left.-\frac{1}{\mu\left(E^{c}\right)} \int_{E^{c}} \int_{I} \mathbf{f}(s, t) d H(t)\right) d \mu(s)\right]\right)
\end{aligned}
$$

$$
\begin{aligned}
& \leq W_{E} \varphi\left(\frac { 1 } { L } \left(\int_{a}^{b} \mathbf{g}(t) d H(t)-\frac{1}{\mu(E)}\right.\right. \\
& \left.\left.\cdot \int_{E} \int_{I} \mathbf{f}(s, t) d H(t)\right) d \mu(s)\right) \\
& +W_{E^{c}} \varphi\left(\frac { 1 } { L } \left(\int_{a}^{b} \mathbf{g}(t) d H(t)-\frac{1}{\mu\left(E^{c}\right)}\right.\right. \\
& \left.\left.\cdot \int_{E^{c}} \int_{I} \mathbf{f}(s, t) d H(t)\right) d \mu(s)\right) \\
& =F(\mathbf{f}, \mathbf{g}, \varphi ; E)
\end{aligned}
$$

for any $E$, which proves the first inequality in (37). By inequality (31), we also have

$$
\begin{aligned}
& F(\mathbf{f}, \mathbf{g}, \varphi ; E)=W_{E} \varphi\left(\frac { 1 } { L } \left(\int_{a}^{b} \mathbf{g}(t) d H(t)\right.\right. \\
& \left.\left.-\frac{1}{\mu(E)} \int_{I} \int_{E} \mathbf{f}(s, t) d \mu(s) d H(t)\right)\right) \\
& +W_{E^{c}} \varphi\left(\frac { 1 } { L } \left(\int_{a}^{b} \mathbf{g}(t) d H(t)\right.\right. \\
& \left.\left.-\frac{1}{\mu\left(E^{c}\right)} \int_{I} \int_{E^{c}} \mathbf{f}(s, t) d \mu(s) d H(t)\right)\right) \\
& \quad \leq W_{E}\left[\frac { 1 } { L } \left(\int_{a}^{b} \varphi(\mathbf{g}(t)) d H(t)\right.\right. \\
& \left.\left.-\frac{1}{\mu(E)} \int_{I} \int_{E} \varphi(\mathbf{f}(s, t)) d \mu(s) d H(t)\right)\right] \\
& +W_{E^{c}}\left[\frac { 1 } { L } \left(\int_{a}^{b} \varphi(\mathbf{g}(t)) d H(t)\right.\right. \\
& \left.\left.\quad-\frac{1}{\mu\left(E^{c}\right)} \int_{I} \int_{E^{c}} \varphi(\mathbf{f}(s, t)) d \mu(s) d H(t)\right)\right] \\
& \quad=\frac{1}{L}\left(\int_{a}^{b} \varphi(\mathbf{g}(t)) d H(t)-\frac{1}{\mu(X)} \varphi(\mathbf{f}(s, t)) d \mu(s) d H(t)\right) \\
& \left.+\int_{I}\right)
\end{aligned}
$$

for any $E$, which proves the second inequality in (37). 
Remark 18. Direct consequences of the previous theorem are the following two inequalities:

$$
\begin{aligned}
& \varphi\left(\frac { 1 } { L } \left(\int_{a}^{b} \mathbf{g}(t) d H(t)\right.\right. \\
& \left.\left.\quad-\frac{1}{\mu(X)} \int_{I} \int_{X} \mathbf{f}(s, t) d \mu(s) d H(t)\right)\right) \\
& \quad \leq \inf _{\{E: 0<\mu(E)<\mu(X)\}} F(\mathbf{f}, \mathbf{g}, \varphi ; E), \\
& \quad \sup _{\{E: 0<\mu(E)<\mu(X)\}} F(\mathbf{f}, \mathbf{g}, \varphi ; E) \leq \frac{1}{L}\left(\int_{a}^{b} \varphi(\mathbf{g}(t)) d H(t)\right. \\
& \left.\quad-\frac{1}{\mu(X)} \int_{I} \int_{X} \varphi(\mathbf{f}(s, t)) d \mu(s) d H(t)\right) .
\end{aligned}
$$

The special case of Theorem 17 can be found in [14] which may be stated as follows.

Corollary 19. Under the assumptions of Corollary 16, the following refinement is valid for every continuous convex function $\varphi: J \rightarrow \mathbb{R}:$

$$
\begin{aligned}
& \varphi\left(\frac { 1 } { L } \left(\int_{a}^{b} g(t) d H(t)\right.\right. \\
& \left.\left.-\frac{1}{\mu(X)} \int_{I} \int_{X} f(s, t) d \mu(s) d H(t)\right)\right) \leq F(f, g, \varphi ; \\
& E) \leq \frac{1}{L}\left(\int_{a}^{b} \varphi(g(t)) d H(t)-\frac{1}{\mu(X)}\right. \\
& \left.\cdot \int_{I} \int_{X} \varphi(f(s, t)) d \mu(s) d H(t)\right)
\end{aligned}
$$

where

$$
\begin{gathered}
F(f, g, \varphi ; E)=W_{E} \varphi\left(\frac { 1 } { L } \left(\int_{a}^{b} g(t) d H(t)\right.\right. \\
\left.\left.-\frac{1}{\mu(E)} \int_{I} \int_{E} f(s, t) d \mu(s) d H(t)\right)\right) \\
+W_{E^{c}} \varphi\left(\frac { 1 } { L } \left(\int_{a}^{b} g(t) d H(t)\right.\right. \\
\left.\left.-\frac{1}{\mu\left(E^{c}\right)} \int_{I} \int_{E^{c}} f(s, t) d \mu(s) d H(t)\right)\right) .
\end{gathered}
$$

4.1. Applications. Haluška and Hutník discussed a class of generalized weighted quasiarithmetic means in the integral form $M_{[a, b], g}(w, f)$ using the integral form of Jensen's inequality [15]. In their work, they used the definition of quasiarithmetic nonsymmetrical weighted mean proposed by Feng [16] which we state below.

Let $[a, b] \subset \mathbb{R}$, where $a<b$, be an interval. Let $L_{1}([a, b])$ denote the vector space of all real Lebesgue measurable functions defined on the interval $[a, b]$ with the classical
Lebesgue measure, and let $L_{1}^{+}([a, b])$ denote the positive cone of $L_{1}([a, b])$, that is, the vector space of all real positive Lebesgue integrable functions on $[a, b]$. Let $\|w\|_{[a, b]}$ denote the finite $L_{1}$-norm of a function $w \in L_{1}^{+}([a, b])$.

Definition 20. Let $(w, f) \in L_{1}^{+}([a, b]) \times L_{1}^{+}([a, b])$ and $g$ : $[0, \infty] \rightarrow \mathbb{R}$ be a real continuous and strictly monotone function. The generalized weighted quasiarithmetic mean of a function $f$ with respect to weight function $w$ is a number $M_{[a, b], g}(w, f) \in \mathbb{R}$, where

$$
\begin{aligned}
& M_{[a, b], g}(w, f) \\
& \quad=g^{-1}\left(\frac{1}{\|w\|_{[a, b]}} \int_{a}^{b} w(x) g(f(x)) d x\right),
\end{aligned}
$$

where $g^{-1}$ denotes the inverse of the function $g$.

In what follows, $g$ is always a real continuous and strictly monotone function (in accordance with Definition 20). Means $M_{[a, b], g}(w, f)$ include many commonly used twovariable integral means as particular cases when taking the suitable functions $w, f$, and $g$. For instance,

(a) for $g(x)=x=I(x)$ (the identity function), we obtain the weighted arithmetic mean:

$$
\begin{aligned}
M_{[a, b], g}(w, f) & =A_{[a, b]}(w, f) \\
& =\frac{1}{\|w\|_{[a, b]}} \int_{a}^{b} w(x) f(x) d x ;
\end{aligned}
$$

(b) for $g(x)=x^{-1}$, we have the weighted harmonic mean:

$$
\begin{aligned}
M_{[a, b], g}(w, f) & =H_{[a, b]}(w, f) \\
& =\left(\frac{1}{\|w\|_{[a, b]}} \int_{a}^{b} \frac{w(x)}{f(x)} d x\right)^{-1}
\end{aligned}
$$

(c) for $g(x)=x^{r}$, we get the weighted power mean of order $r$ :

$$
\begin{aligned}
& M_{[a, b], g}(w, f)=M^{[r]}(f ; w ; a, b) \\
& =\left\{\begin{array}{l}
\left(\frac{1}{\|w\|_{[a, b]}} \int_{a}^{b} w(x) f(x)^{r} d x\right)^{1 / r} ; \\
\exp \left(\frac{1}{\|w\|_{[a, b]}} \int_{a}^{b} w(x) \ln f(x) d x\right) .
\end{array}\right.
\end{aligned}
$$

The case $r=0$ corresponds to the weighted geometric mean.

Under the assumptions of Corollary 19, we define the following notations where $S \in\left\{X, E, E^{\mathcal{C}}\right\}$. Throughout this section, we also assume that $\ln$ and exp have the natural domain. 
Arithmetic Mean. It is as follows:

$$
\begin{aligned}
& A_{g}^{\prime}=\frac{1}{L} \int_{a}^{b} g(t) d H(t) \\
& A_{S}=\frac{1}{L \cdot \mu(S)} \int_{I} \int_{S} f(s, t) d \mu(s) d H(t) \\
& \widetilde{A}_{S}=A_{g}^{\prime}-A_{S} .
\end{aligned}
$$

Geometric Mean. It is as follows:

$$
\begin{aligned}
& G_{g}^{\prime}=\exp \left(\frac{1}{L} \int_{a}^{b} \ln g(t) d H(t)\right) \\
& G_{S}=\exp \left(\frac{1}{L \cdot \mu(S)} \int_{I} \int_{S} \ln f(s, t) d \mu(s) d H(t)\right) \\
& \widetilde{G}_{S}=\frac{G_{g}^{\prime}}{G_{S}}
\end{aligned}
$$

Harmonic Mean. It is as follows:

$$
\begin{aligned}
H_{g}^{\prime} & =\left(\frac{1}{L} \int_{a}^{b} \frac{1}{g(t)} d H(t)\right)^{-1}, \\
H_{S} & =\left(\frac{1}{L \cdot \mu(S)} \int_{I} \int_{S} \frac{1}{f(s, t)} d \mu(s) d H(t)\right)^{-1}, \\
\frac{1}{\widetilde{H}_{S}} & =\frac{1}{H_{g}^{\prime}}-\frac{1}{H_{S}} .
\end{aligned}
$$

Power Mean. It is as follows:

$$
\begin{aligned}
M_{g}^{\prime} & =\frac{1}{L} \int_{a}^{b} \mathbf{g}(t)^{r} d H(t), \\
M_{S}^{[r]} & =\frac{1}{L \cdot \mu(S)} \int_{I} \int_{S} \mathbf{f}(s, t)^{r} d \mu(s) d H(t), \\
\widetilde{M}_{S}^{[r]} & =\left(M_{g}^{\prime}-M_{S}^{[r]}\right)^{1 / r} .
\end{aligned}
$$

We now define a relationship between arithmetic and geometric means.

Theorem 21. Consider $\widetilde{G}_{X} \leq \widetilde{A}_{E}^{W_{E}} \cdot \widetilde{A}_{E^{c}}^{W_{E^{c}}} \leq \widetilde{A}_{X}$.
Proof. In (43), let $\varphi(x)=-\ln (x)$ to get

$$
\begin{aligned}
&- \ln \left(\frac{1}{L} \int_{a}^{b} g(t) d H(t)\right. \\
&-\left.\frac{1}{L \cdot \mu(X)} \int_{I} \int_{X} f(s, t) d \mu(s) d H(t)\right) \leq-W_{E} \\
& \cdot \ln \left(\frac{1}{L} \int_{a}^{b} g(t) d H(t)\right. \\
&-\left.\frac{1}{L \cdot \mu(E)} \int_{I} \int_{E} f(s, t) d \mu(s) d H(t)\right)-W_{E^{c}} \\
& \cdot \ln \left(\frac{1}{L} \int_{a}^{b} g(t) d H(t)\right. \\
&-\left.\frac{1}{L \cdot \mu\left(E^{c}\right)} \int_{I} \int_{E^{c}} f(s, t) d \mu(s) d H(t)\right) \leq-\left(\frac{1}{L}\right. \\
& \cdot \int_{a}^{b} \ln (g(t)) d H(t)-\frac{1}{L \cdot \mu(X)} \\
&\left.\cdot \int_{I} \int_{X} \ln (f(s, t)) d \mu(s) d H(t)\right) .
\end{aligned}
$$

In our notation, we have

$$
\begin{aligned}
& -\ln \left(A_{g}^{\prime}-A_{X}\right) \leq-W_{E} \ln \left(A_{g}^{\prime}-A_{E}\right)-W_{E^{c}} \ln \left(A_{g}^{\prime}\right. \\
& \left.-A_{E^{c}}\right) \leq-\left[\ln \left(\exp \frac{1}{L} \int_{a}^{b} \ln (g(t)) d H(t)\right)\right. \\
& -\ln \left(\exp \frac{1}{L \cdot \mu(X)}\right. \\
& \left.\left.\cdot \int_{I} \int_{X} \ln (f(s, t)) d \mu(s) d H(t)\right)\right] .
\end{aligned}
$$

Further simplification gives us

$$
\begin{aligned}
-\ln \widetilde{A}_{X} & \leq-\left(W_{E} \ln \widetilde{A}_{E}+W_{E^{c}} \ln \widetilde{A}_{E^{c}}\right) \\
& \leq-\left[\ln G_{g}^{\prime}-\ln G_{X}\right] .
\end{aligned}
$$

Using the property of $\ln$ gives us

$$
-\ln \widetilde{A}_{X} \leq-\left(\ln \widetilde{A}_{E}^{W_{E}}+\ln \widetilde{A}_{E^{c}}^{W_{E}^{c}}\right) \leq-\ln \widetilde{G}_{X}
$$

which can be written as

$$
\ln \widetilde{A}_{X} \geq \ln \left(\widetilde{A}_{E}^{W_{E}} \cdot \widetilde{A}_{E^{c}}^{W_{c}^{c}}\right) \geq \ln \widetilde{G}_{X} .
$$

Finally,

$$
\widetilde{G}_{X} \leq \widetilde{A}_{E}^{W_{E}} \cdot \widetilde{A}_{E^{c}}^{W_{E^{c}}} \leq \widetilde{A}_{X}
$$

Here we obtain another relationship between geometric and arithmetic means. 
Theorem 22. Consider $\widetilde{G}_{X} \leq W_{E} \widetilde{G}_{E}+W_{E^{c}} \widetilde{G}_{E^{c}} \leq \widetilde{A}_{X}$.

Proof. Take $\varphi(x)=\exp (x)$; if we replace $g(t)$ with $\ln g(t)$ and $f(s, t)$ with $\ln f(s, t)$ in (43), then we get

$$
\begin{aligned}
& \exp \left(\frac{1}{L} \int_{a}^{b} \ln g(t) d H(t)\right. \\
& \left.-\frac{1}{L \cdot \mu(X)} \int_{I} \int_{X} \ln f(s, t) d \mu(s) d H(t)\right) \leq W_{E} \\
& \cdot \exp \left(\frac{1}{L} \int_{a}^{b} \ln g(t) d H(t)\right. \\
& \left.-\frac{1}{L \cdot \mu(E)} \int_{I} \int_{E} \ln f(s, t) d \mu(s) d H(t)\right)+W_{E^{c}} \\
& \cdot \exp \left(\frac{1}{L} \int_{a}^{b} \ln g(t) d H(t)\right. \\
& \left.-\frac{1}{L \cdot \mu\left(E^{c}\right)} \int_{I} \int_{E^{c}} \ln f(s, t) d \mu(s) d H(t)\right) \leq \frac{1}{L} \\
& \cdot \int_{a}^{b} \exp (\ln g(t)) d H(t)-\frac{1}{L \cdot \mu(X)} \\
& \cdot \int_{I} \int_{X} \exp (\ln f(s, t)) d \mu(s) d H(t) .
\end{aligned}
$$

Using the property of exp, we have

$$
\begin{aligned}
& \frac{\exp \left((1 / L) \int_{a}^{b} \ln g(t) d H(t)\right)}{\exp \left((1 /(L \cdot \mu(X))) \int_{I} \int_{X} \ln f(s, t) d \mu(s) d H(t)\right)} \\
& \leq W_{E} \\
& \cdot \frac{\exp \left((1 / L) \int_{a}^{b} \ln g(t) d H(t)\right)}{\exp \left((1 /(L \cdot \mu(E))) \int_{I} \int_{E} \ln f(s, t) d \mu(s) d H(t)\right)} \\
& +W_{E^{c}} \frac{\exp \left((1 / L) \int_{a}^{b} \ln g(t) d H(t)\right)}{\exp \left(\left(1 /\left(L \cdot \mu\left(E^{c}\right)\right)\right) \int_{I} \int_{E^{c}} \ln f(s, t) d \mu(s) d H(t)\right)} \\
& \leq \frac{1}{L} \int_{a}^{b} g(t) d H(t)-\frac{1}{L \cdot \mu(X)} \\
& \cdot \int_{I} \int_{X} f(s, t) d \mu(s) d H(t) .
\end{aligned}
$$

In out notations, we have

$$
\frac{G_{g}^{\prime}}{G_{X}} \leq W_{E} \frac{G_{g}^{\prime}}{G_{E}}+W_{E^{c}} \frac{G_{g}^{\prime}}{G_{E^{c}}} \leq A_{g}^{\prime}-A_{X} .
$$

Finally,

$$
\widetilde{G}_{X} \leq W_{E} \widetilde{G}_{E}+W_{E^{c}} \widetilde{G}_{E^{c}} \leq \widetilde{A}_{X}
$$

The following theorem states a relationship between geometric and harmonic means.

Theorem 23. Consider $1 / \widetilde{G}_{X} \leq 1 / \widetilde{H}_{E}^{W_{E}} \widetilde{H}_{E^{c}}^{W_{E^{c}}} \leq 1 / \widetilde{H}_{X}$.

Proof. Take $\varphi(x)=-\ln (x)$; replace $g(t)$ with $1 / g(t)$ and $f(s, t)$ with $1 / f(s, t)$ in (43) to get

$$
\begin{aligned}
& -\ln \left(\frac{1}{L} \int_{a}^{b} \frac{1}{g(t)} d H(t)\right. \\
& \left.-\frac{1}{L \cdot \mu(X)} \int_{I} \int_{X} \frac{1}{f(s, t)} d \mu(s) d H(t)\right) \leq-W_{E} \\
& \cdot \ln \frac{1}{L}\left(\int_{a}^{b} \frac{1}{g(t)} d H(t)-\frac{1}{\mu(E)}\right. \\
& \left.\cdot \int_{I} \int_{E} \frac{1}{f(s, t)} d \mu(s) d H(t)\right)-W_{E^{c}} \ln \frac{1}{L} \\
& \cdot\left(\int_{a}^{b} \frac{1}{g(t)} d H(t)-\frac{1}{\mu\left(E^{c}\right)}\right. \\
& \left.\cdot \int_{I} \int_{E^{c}} \frac{1}{f(s, t)} d \mu(s) d H(t)\right) \leq-\frac{1}{L} \\
& \cdot \int_{a}^{b} \ln \left(\frac{1}{g(t)}\right) d H(t)+\frac{1}{L \cdot \mu(X)} \\
& \cdot \int_{I} \int_{X} \ln \left(\frac{1}{f(s, t)}\right) d \mu(s) d H(t) .
\end{aligned}
$$

In our notations, we have

$$
\begin{aligned}
& -\ln \left[\left(H_{g}^{\prime}\right)^{-1}-\left(H_{X}\right)^{-1}\right] \leq-W_{E} \\
& \cdot \ln \left[\left(H_{g}^{\prime}\right)^{-1}-\left(H_{E}\right)^{-1}\right]-W_{E^{c}} \\
& \cdot \ln \left[\left(H_{g}^{\prime}\right)^{-1}-\left(H_{E^{c}}\right)^{-1}\right] \\
& \leq-\left(\frac{1}{L} \int_{a}^{b} \ln 1-\ln g(t) d H(t)\right. \\
& \left.-\frac{1}{L \cdot \mu(X)} \int_{I} \int_{X} \ln 1-\ln f(s, t) d \mu(s) d H(t)\right)
\end{aligned}
$$

which gives us

$$
\begin{gathered}
-\ln \left(\widetilde{H}_{X}\right)^{-1} \leq-W_{E} \ln \left(\widetilde{H}_{E}\right)^{-1}-W_{E^{c}} \ln \left(\widetilde{H}_{E^{c}}\right)^{-1} \\
\leq-\left(-\frac{1}{L} \int_{a}^{b} \ln g(t) d H(t)\right. \\
\left.+\frac{1}{L \cdot \mu(X)} \int_{I} \int_{X} \ln f(s, t) d \mu(s) d H(t)\right) .
\end{gathered}
$$


Multiplying the last inequality above by $\ln$ exp, we get

$$
\begin{aligned}
& -\ln \frac{1}{\left(\widetilde{H}_{X}\right)} \leq-W_{E} \ln \frac{1}{\left(\widetilde{H}_{E}\right)}-W_{E^{c}} \ln \frac{1}{\left(\widetilde{H}_{E^{c}}\right)} \\
& \quad \leq-\left(-\ln \exp \frac{1}{L} \int_{a}^{b} \ln g(t) d H(t)\right. \\
& \left.\quad+\ln \exp \frac{1}{L \cdot \mu(X)} \int_{I} \int_{X} \ln f(s, t) d \mu(s) d H(t)\right),
\end{aligned}
$$

which can be written as

$$
\begin{aligned}
-\ln \frac{1}{\left(\widetilde{H}_{X}\right)} & \leq-\ln \left(\frac{1}{\widetilde{H}_{E}}\right)^{W_{E}}-\ln \left(\frac{1}{\widetilde{H}_{E^{c}}}\right)^{W_{E^{c}}} \\
& \leq-\left[-\ln G_{g}^{\prime}+\ln \left(G_{X}\right)\right] .
\end{aligned}
$$

Using the property of $\ln$, we have

$$
-\ln \frac{1}{\left(\widetilde{H}_{X}\right)} \leq-\left(\ln \frac{1}{\widetilde{H}_{E}^{W_{E}}}+\ln \frac{1}{\widetilde{H}_{E^{c}}^{W_{E^{c}}}}\right) \leq-\ln \frac{\left(G_{X}\right)}{G_{g}^{\prime}}
$$

Simplifying the above, we get

$$
-\ln \frac{1}{\left(\widetilde{H}_{X}\right)} \leq-\ln \left(\frac{1}{\widetilde{H}_{E}^{W_{E}}} \frac{1}{\widetilde{H}_{E^{c}}^{W_{E^{c}}}}\right) \leq-\ln \frac{1}{\widetilde{G}_{X}} .
$$

Finally, we get

$$
\frac{1}{\widetilde{G}_{X}} \leq \frac{1}{\widetilde{H}_{E}^{W_{E}} \widetilde{H}_{E^{c}}^{W_{E^{c}}}} \leq \frac{1}{\widetilde{H}_{X}}
$$

Now we define another relationship between geometric and harmonic means.

Theorem 24. Consider $1 / \widetilde{G}_{X} \leq W_{E} / \widetilde{G}_{E}+W_{E^{c}} / \widetilde{G}_{E^{c}} \leq 1 / \widetilde{H}_{X}$.

Proof. In (43), take $\varphi(x)=\exp (x)$ and replace $g(t)$ with $\ln (1 / g(t))$ and $f(s, t)$ with $\ln (1 / f(s, t))$ to get

$$
\begin{aligned}
& \exp \left(\frac{1}{L} \int_{a}^{b} \ln \frac{1}{g(t)} d H(t)\right. \\
& \left.-\frac{1}{L \cdot \mu(X)} \int_{I} \int_{X} \ln \frac{1}{f(s, t)} d \mu(s) d H(t)\right) \leq W_{E} \\
& \cdot \exp \left(\frac{1}{L} \int_{a}^{b} \ln \frac{1}{g(t)} d H(t)\right. \\
& \left.-\frac{1}{L \cdot \mu(E)} \int_{I} \int_{E} \ln \frac{1}{f(s, t)} d \mu(s) d H(t)\right)+W_{E^{c}}
\end{aligned}
$$

$$
\begin{aligned}
& \cdot \exp \left(\frac{1}{L} \int_{a}^{b} \ln \frac{1}{g(t)} d H(t)\right. \\
& \left.-\frac{1}{L \cdot \mu\left(E^{c}\right)} \int_{I} \int_{E^{c}} \ln \frac{1}{f(s, t)} d \mu(s) d H(t)\right) \\
& \leq \frac{1}{L}\left(\int_{a}^{b} \exp \left(\ln \frac{1}{g(t)}\right) d H(t)-\frac{1}{\mu(X)}\right. \\
& \left.\cdot \int_{I} \int_{X} \exp \left(\ln \frac{1}{f(s, t)}\right) d \mu(s) d H(t)\right) .
\end{aligned}
$$

Using the property of ln, we have

$$
\begin{aligned}
& \exp \left(\frac{1}{L} \int_{a}^{b}-\ln g(t) d H(t)\right. \\
& \left.+\frac{1}{L \cdot \mu(X)} \int_{I} \int_{X} \ln f(s, t) d \mu(s) d H(t)\right) \leq W_{E} \\
& \cdot \exp \left(\frac{1}{L} \int_{a}^{b}-\ln g(t) d H(t)\right. \\
& \left.+\frac{1}{L \cdot \mu(E)} \int_{I} \int_{\mathrm{E}} \ln f(s, t) d \mu(s) d H(t)\right)+W_{E^{c}} \\
& \cdot \exp \left(\frac{1}{L} \int_{a}^{b}-\ln g(t) d H(t)\right. \\
& \left.+\frac{1}{L \cdot \mu\left(E^{c}\right)} \int_{I} \int_{E^{c}} \ln \frac{1}{f(s, t)} d \mu(s) d H(t)\right) \leq \frac{1}{L} \\
& \cdot \int_{a}^{b} \frac{1}{g(t)} d H(t)-\frac{1}{L \cdot \mu(X)} \\
& . \int_{I} \int_{X} \frac{1}{f(s, t)} d \mu(s) d H(t) .
\end{aligned}
$$

Using the property of exp, we have

$$
\begin{aligned}
& \frac{\exp \left((1 /(L \cdot \mu(X))) \int_{I} \int_{X} \ln f(s, t) d \mu(s) d H(t)\right)}{\exp \left((1 / L) \int_{a}^{b} \ln g(t) d H(t)\right)} \\
& \leq W_{E} \\
& +\frac{\exp \left((1 /(L \cdot \mu(E))) \int_{I} \int_{E} \ln f(s, t) d \mu(s) d H(t)\right)}{\exp \left((1 / L) \int_{a}^{b} \ln g(t) d H(t)\right)} \\
& +W_{E^{c}} \\
& \quad \frac{\exp \left(\left(1 /\left(L \cdot \mu\left(E^{c}\right)\right)\right) \int_{I} \int_{E^{c}} \ln f(s, t) d \mu(s) d H(t)\right)}{\quad \exp \left((1 / L) \int_{a}^{b} \ln g(t) d H(t)\right)} \\
& \leq \frac{1}{H_{g}^{\prime}}-\frac{1}{H_{X}} .
\end{aligned}
$$


In our notations, we have

$$
\frac{G_{X}}{G_{g}^{\prime}} \leq W_{E} \frac{G_{E}}{G_{g}^{\prime}}+W_{E^{c}} \frac{G_{E^{c}}}{G_{g}^{\prime}} \leq \frac{1}{\widetilde{H}_{X}} .
$$

Finally,

$$
\frac{1}{\widetilde{G}_{X}} \leq \frac{W_{E}}{\widetilde{G}_{E}}+\frac{W_{E^{c}}}{\widetilde{G}_{E^{c}}} \leq \frac{1}{\widetilde{H}_{X}} .
$$

Now we define a relationship between power mean and arithmetic mean.

Theorem 25. (i) For $r \leq 1$, we have

$$
\widetilde{M}_{X}^{[r]} \leq W_{E} \widetilde{M}_{E}^{[r]}+W_{E^{c}}^{[r]} \widetilde{M}_{E^{c}} \leq \widetilde{A}_{X} .
$$

(ii) For $r \geq 1$, the above inequalities are reversed.

Proof. (i) In (43), take $\varphi(x)=x^{1 / r}$ and replace $g(t)$ with $(g(t))^{r}$ and $f(s, t)$ with $(f(s, t))^{r}$ to get

$$
\begin{aligned}
& \left(\frac{1}{L} \int_{a}^{b}(g(t))^{r} d H(t)\right. \\
& \left.-\frac{1}{L \cdot \mu(X)} \int_{I} \int_{X} f(s, t) d \mu(s) d H(t)\right)^{1 / r} \\
& \quad \leq W_{E}\left(\frac{1}{L} \int_{a}^{b}(g(t))^{r} d H(t)\right. \\
& \left.-\frac{1}{L \cdot \mu(E)} \int_{I} \int_{E}(f(s, t))^{r} d \mu(s) d H(t)\right)^{1 / r} \\
& +W_{E^{c}}\left(\frac{1}{L} \int_{a}^{b}(g(t))^{r} d H(t)\right. \\
& \left.-\frac{1}{L \cdot \mu\left(E^{c}\right)} \int_{I} \int_{E^{c}}(f(s, t))^{r} d \mu(s) d H(t)\right)^{1 / r} \\
& \quad \leq \frac{1}{L} \int_{a}^{b}\left((g(t))^{r}\right)^{1 / r} d H(t)-\frac{1}{L \cdot \mu(X)} \\
& \quad \cdot \int_{I} \int_{X}\left((f(s, t))^{r}\right)^{1 / r} d \mu(s) d H(t) .
\end{aligned}
$$

In out notations, we have

$$
\begin{aligned}
\left(M_{g}^{\prime}-M_{X}^{[r]}\right)^{1 / r} \\
\leq W_{E}\left(M_{g}^{\prime}-M_{E}^{[r]}\right)^{1 / r}+W_{E^{c}}\left(M_{g}^{\prime}-M_{E^{c}}^{[r]}\right)^{1 / r} \\
\leq \frac{1}{L} \int_{a}^{b} g(t) d H(t) \\
\quad-\frac{1}{L \cdot \mu(X)} \int_{I} \int_{X} f(s, t) d \mu(s) d H(t),
\end{aligned}
$$

which can be written as

$$
\begin{aligned}
\left(M_{g}^{\prime}-M_{X}^{[r]}\right)^{1 / r} \leq & W_{E}\left(M_{g}^{\prime}-M_{E}^{[r]}\right)^{1 / r} \\
& +W_{E^{c}}\left(M_{g}^{\prime}-M_{E^{c}}^{[r]}\right)^{1 / r} \\
\leq & \left(A_{g}^{\prime}-A_{X}\right) .
\end{aligned}
$$

Finally,

$$
\widetilde{M}_{X}^{[r]} \leq W_{E} \widetilde{M}_{E}^{[r]}+W_{E^{c}} \widetilde{M}_{E^{c}}^{[r]} \leq \widetilde{A}_{X} .
$$

(ii) If $r \geq 1$, then $\varphi(x)=x^{1 / r}$ is concave, so the inequalities in (76) are reversed.

The following theorem gives another relationship between power and arithmetic means.

Theorem 26. Let $r, s \in \mathbb{R}, r \leq s$.

(i) If $s \geq 0$, then

$$
\left(\widetilde{M}_{X}^{[r]}\right)^{s} \leq W_{E}\left(\widetilde{M}_{E}\right)^{s}+W_{E^{c}}\left(\widetilde{M}_{E^{c}}\right)^{s} \leq\left(\widetilde{A}_{X}\right)^{s} .
$$

(ii) For $s<0$, the above inequalities are reversed.

Proof. (i) In (43), take $\varphi(x)=x^{s / r}$ and replace $g(t)$ with $(g(t))^{r}$ and $f(s, t)$ with $(f(s, t))^{r}$ to get

$$
\begin{aligned}
& \left(\frac{1}{L} \int_{a}^{b}(g(t))^{r} d H(t)\right. \\
& \left.\quad-\frac{1}{L \cdot \mu(X)} \int_{I} \int_{X} f(s, t) d \mu(s) d H(t)\right)^{s / r} \\
& \quad \leq W_{E}\left(\frac{1}{L} \int_{a}^{b}(g(t))^{r} d H(t)\right. \\
& \left.-\frac{1}{L \cdot \mu(E)} \int_{I} \int_{E}(f(s, t))^{r} d \mu(s) d H(t)\right)^{s / r} \\
& +W_{E^{c}}\left(\frac{1}{L} \int_{a}^{b}(g(t))^{r} d H(t)\right. \\
& \left.\quad-\frac{1}{L \cdot \mu\left(E^{c}\right)} \int_{I} \int_{E^{c}}(f(s, t))^{r} d \mu(s) d H(t)\right)^{s / r} \\
& \quad \leq \frac{1}{L}\left(\int_{a}^{b}\left((g(t))^{r}\right)^{s / r} d H(t)\right. \\
& \left.\quad-\frac{1}{\mu(X)} \int_{I} \int_{X}\left((f(s, t))^{r}\right)^{s / r} d \mu(s) d H(t)\right) .
\end{aligned}
$$


In our notations, we have

$$
\begin{aligned}
\left(M_{g}^{\prime}-M_{X}^{[r]}\right)^{s / r} \\
\leq W_{E}\left(M_{g}^{\prime}-M_{E}^{[r]}\right)^{s / r}+W_{E^{c}}\left(M_{g}^{\prime}-M_{E^{c}}^{[r]}\right)^{s / r} \\
\leq \frac{1}{L} \int_{a}^{b} g(t)^{s} d H(t) \\
\quad-\frac{1}{L \cdot \mu(X)} \int_{I} \int_{X} f(s, t)^{s} d \mu(s) d H(t) .
\end{aligned}
$$

Further simplification gives us

$$
\begin{aligned}
\left(M_{g}^{\prime}-M_{X}^{[r]}\right)^{s / r} \leq & W_{E}\left(M_{g}^{\prime}-M_{E}^{[r]}\right)^{s / r} \\
& +W_{E^{c}}\left(M_{g}^{\prime}-M_{E^{c}}^{[r]}\right)^{s / r} \\
\leq & \left(A_{g}^{\prime}-A_{X}\right)^{s} .
\end{aligned}
$$

Finally,

$$
\left(\widetilde{M}_{X}^{[r]}\right)^{s} \leq W_{E}\left(\widetilde{M}_{E}\right)^{s}+W_{E^{c}}\left(\widetilde{M}_{E^{c}}\right)^{s} \leq\left(\widetilde{A}_{X}\right)^{s}
$$

(ii) For $s<0$, the function $\varphi(x)=x^{s / r}$ is concave, so the inequalities in (81) are reversed.

\section{Competing Interests}

The authors declare that they have no competing interests.

\section{References}

[1] J. Pečarić, F. Proschan, and Y. L. Tong, Convex Functions, Partial Orderings, and Statistical Applications, Academic Press, New York, NY, USA, 1992.

[2] D. S. Mitrinović, J. E. Pečarić, and A. M. Fink, Classical and New Inequalities in Analysis, Kluwer Academic Publishers, Dordrecht, The Netherlands, 1993.

[3] A. McD Mercer, “A variant of Jensen's inequality," Journal of Inequalities in Pure and Applied Mathematics, vol. 4, article 73, 2003.

[4] S. Abramovich, M. Klaričić Bakula, M. Matić, and J. Pečarić, "A variant of Jensen-Steffensen's inequality and quasi-arithmetic means," Journal of Mathematical Analysis and Applications, vol. 307, no. 1, pp. 370-386, 2005.

[5] W. S. Cheung, A. Matković, and J. Pečarić, "A variant of Jessen's inequality and generalized means," Journal of Inequalities in Pure and Applied Mathematics, vol. 7, no. 1, article 10, 2006.

[6] M. Niezgoda, "A generalization of Mercer's result on convex functions," Nonlinear Analysis: Theory, Methods \& Applications, vol. 71, no. 7-8, pp. 2771-2779, 2009.

[7] J. Pečarić, "A variant of Jensen's inequality," Journal of Inequalities in Pure and Applied Mathematics, vol. 4, no. 4, article 73, 2003.

[8] G. H. Hardy, J. E. Littlewood, and G. Pólya, Inequalities, Cambridge University Press, Cambridge, UK, 1978.

[9] J. E. Pečarić, "On some inequalities for functions with nondecreasing increments," Journal of Mathematical Analysis and Applications, vol. 98, no. 1, pp. 188-197, 1984.
[10] H. D. Brunk, "Integral inequalities for functions with nondecreasing increments," Pacific Journal of Mathematics, vol. 14, pp. 783-793, 1964.

[11] J. E. Pečarić, "An inequality for 3-convex functions," Journal of Mathematical Analysis and Applications, vol. 90, no. 1, pp. 213218, 1982.

[12] J. E. Pečarić, "Generalization of some results of H. Burkill and L. Mirsky and some related results," Periodica Mathematica Hungarica, vol. 15, no. 3, pp. 241-247, 1984.

[13] A. W. Marshall, I. Olkin, and B. C. Arnold, Inequalities: Theory of Majorization and Its Applications, Springer Series in Statistics, Springer, New York, NY, USA, 2nd edition, 2011.

[14] A. R. Khan, J. Pečarić, and M. Praljak, "A note on generalized Mercer's inequality," Bulletin of Malaysian Maths Science Society, 2015.

[15] J. Haluška and O. Hutník, "Some inequalities involving integral means," Tatra Mountains Mathematical Publications, vol. 35, pp. 131-146, 2007.

[16] Q. I. Feng, "Generalized abstracted mean values," Journal of Inequalities in Pure and Applied Mathematics, vol. 1, no. 1, article 4, 2000. 


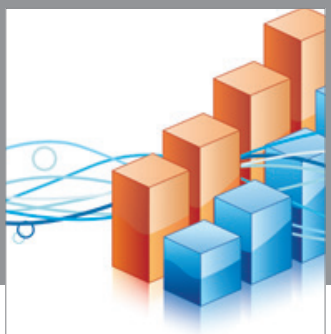

Advances in

Operations Research

vatem alat4

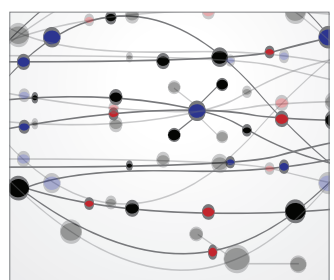

\section{The Scientific} World Journal
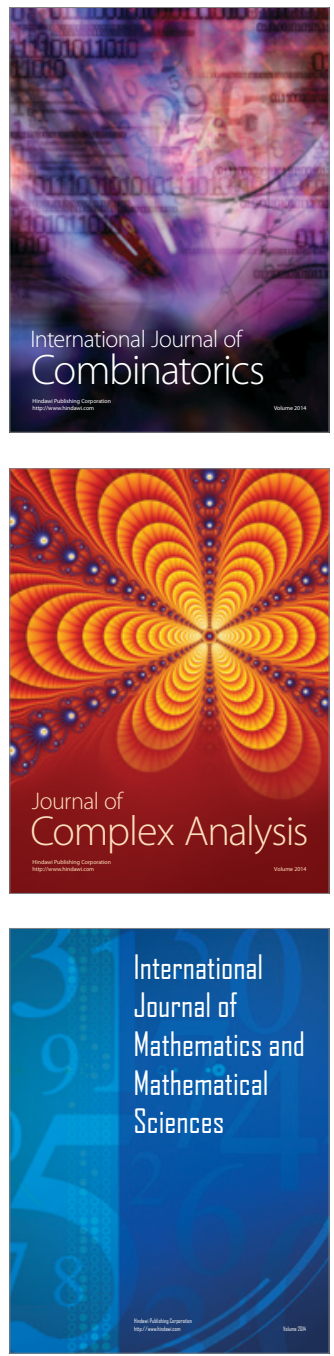
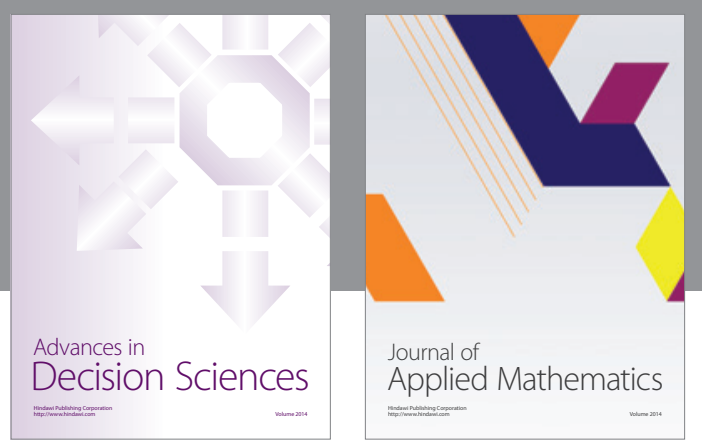

Algebra

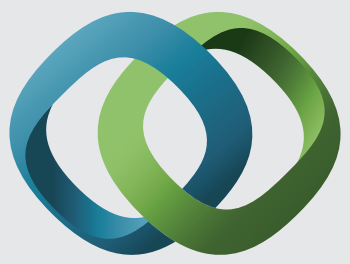

\section{Hindawi}

Submit your manuscripts at

http://www.hindawi.com
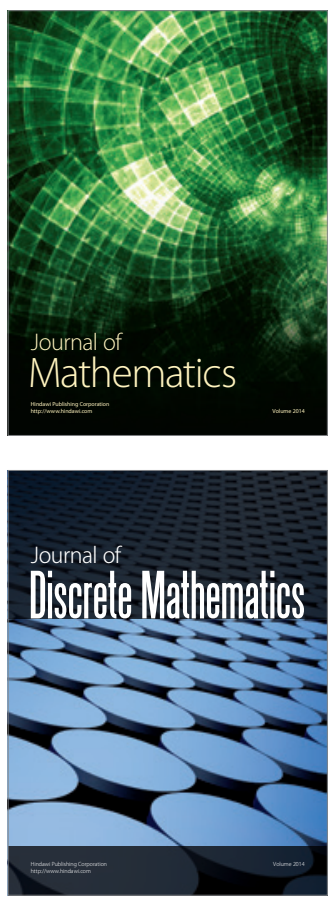

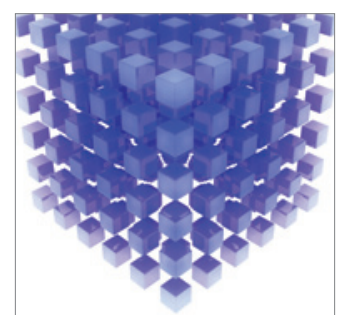

Mathematical Problems in Engineering
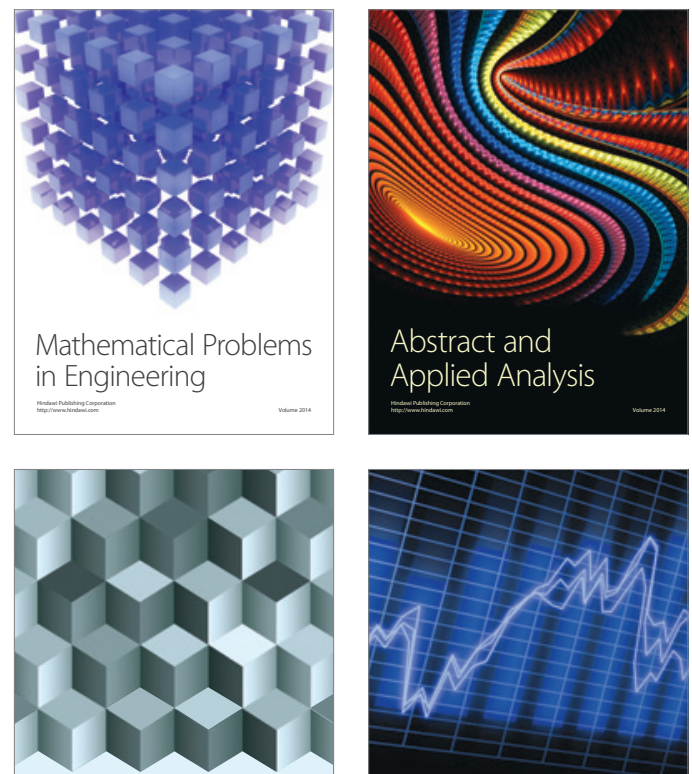

Journal of

Function Spaces

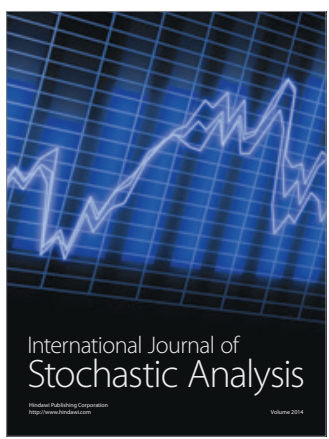

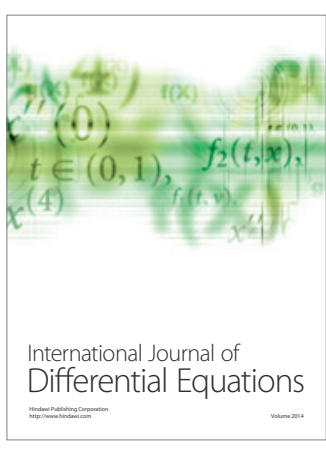
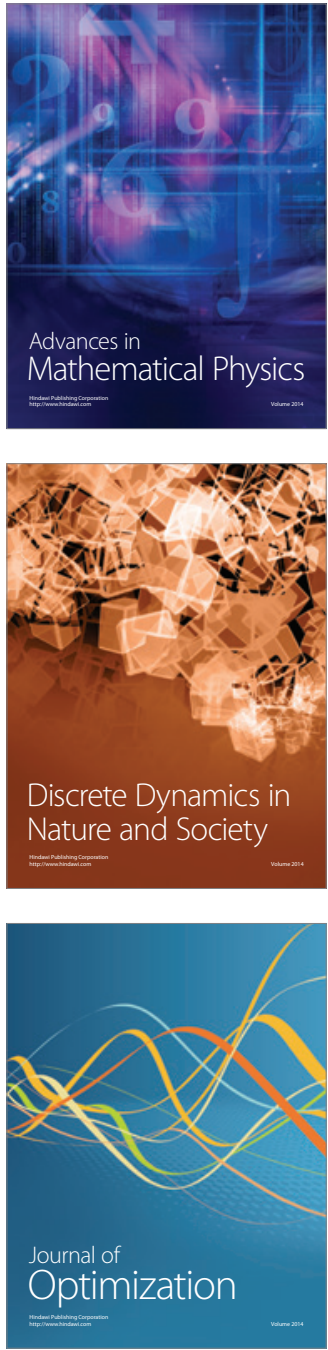